\title{
Emergent Coherent Lattice Behavior in Kondo Nanosystems
}

\author{
Marcin Raczkowski and Fakher F. Assaad \\ Institut für Theoretische Physik und Astrophysik, \\ Universität Würzburg, Am Hubland, D-97074 Würzburg, Germany
}

(Dated: March 12, 2019)

\begin{abstract}
How many magnetic moments periodically arranged on a metallic surface are needed to generate a coherent Kondo lattice behavior? We investigate this fundamental issue within the particle-hole symmetric Kondo lattice model using quantum Monte Carlo simulations. Extra magnetic atoms forming closed shells around the initial impurity induce a fast splitting of the Kondo resonance at the inner shells which signals the formation of composite heavy-fermion bands. The onset of the hybridization gap matches well the enhancement of antiferromagnetic spin correlations in the plane perpendicular to the applied magnetic field, a genuine feature of the coherent Kondo lattice. In contrast, the outermost shell remains dominated by a local Kondo physics with spectral features resembling the single-impurity behavior.
\end{abstract}

In the realm of condensed matter physics, the theory of wave propagation in periodic structures - the Bloch theorem [1] - forms the basis for calculating the electronic band structure of solids. It is also a cornerstone for understanding low-temperature Fermi-liquid-like $\propto T^{2}$ transport properties of heavy-fermion (HF) metals which arise from a coherent Kondo screening of periodically arranged $f$-shell moments by conduction electrons [2]. The quantum entanglement between localized $f$-moments and mobile conduction electrons leads to the enlargement of the Fermi surface, which determines, below the coherence scale $T_{\text {coh }}$, transport and thermodynamic properties of HF liquids. A coherent Kondo lattice behavior differs markedly from the single-impurity Kondo physics with a logarithmic increase, below the Kondo temperature $T_{K}$, of spin-flip scattering for conduction electrons [3]. Elucidating the crossover between both regimes [4-14] as well as identifying the energy scale $T_{\text {coh }}$ associated with the formation of the coherent HF state is a long-standing issue [15-27].

Starting from an incoherent dilute limit of Kondo impurities, one possibility to study how the lattice effects come into play is to increase the concentration of magnetic ions [28-35]. However, the electronic structure of bulk HF materials is essentially three dimensional, and one may wonder how the coherence phenomena are affected either by reduced dimensionality or in spatially restricted geometries where both quantum fluctuations and correlations effect are enhanced $[36,37]$. In this respect, the experimental realization of artificial $f$-electron superlattices [38] has opened a new avenue to investigate the onset of coherence in a two-dimensional (2D) regime followed up by theoretical studies [39].

In recent years, new insight into the Kondo physics at the nanoscale has come from scanning tunneling microscopy (STM) [40, 41]. It allows one to probe Kondo screening at a single magnetic adatom [42, 43], to study the composite nature of the HF quasiparticles in a lattice situation [44-47], and also to image a mutual RKKY interaction between magnetic impurities mediated by con- duction electrons as a function of the interatomic distances [48]. The possibility for a systematic and controlled study of the competition between different energy scales in Kondo nanostructures resulted in a resurgence of interest in the interplay between the Kondo effect and magnetic RKKY correlations in adatom dimers [49-52], trimers [53], and multiple impurities [54-56].

Given the distinct difference between a single-impurity Kondo physics and the coherent Kondo lattice behavior, it is natural to ask how many magnetic moments periodically arranged on a metallic surface are needed to resolve a crossover between both regimes? In this Letter, we address this question on the basis of the Kondo lattice model (KLM) at half-filling [57],

$$
H_{\mathrm{KLM}}=-t \sum_{\langle\boldsymbol{i}, \boldsymbol{j}\rangle, \sigma} c_{\boldsymbol{i}, \sigma}^{\dagger} c_{\boldsymbol{j}, \sigma}+\sum_{\boldsymbol{i}} J_{\boldsymbol{i}} \boldsymbol{S}_{\boldsymbol{i}}^{c} \cdot \boldsymbol{S}_{\boldsymbol{i}}^{f},
$$

where $\boldsymbol{S}_{\boldsymbol{i}}^{c}=\frac{1}{2} \sum_{\sigma, \sigma^{\prime}} c_{\boldsymbol{i}, \sigma^{\dagger}}^{\dagger} \boldsymbol{\sigma}_{\sigma, \sigma^{\prime}} c_{\boldsymbol{i}, \sigma^{\prime}}$ are spin operators of conduction electrons and $\boldsymbol{S}_{\boldsymbol{i}}^{f}=\frac{1}{2} \sum_{\sigma, \sigma^{\prime}} f_{\boldsymbol{i}, \sigma}^{\dagger} \boldsymbol{\sigma}_{\sigma, \sigma^{\prime}} f_{\boldsymbol{i}, \sigma^{\prime}}$ are localized spins with $\sigma$ being the Pauli matrices. The Hamiltonian (1) describes localized spin 1/2 magnetic moments coupled via site-dependent antiferromagnetic (AF) exchange interaction $J_{i}$ to conduction electrons on a square lattice. By switching on and off the values of $J_{\boldsymbol{i}}$, we can investigate a crossover from the zero-dimensional Kondo effect to the 2D particle-hole symmetric Kondo lattice, where the coherence of individual Kondo screening clouds opens up a hybridization gap at the Fermi level and gives rise to the Kondo insulating phase. We study the most challenging regime $J / t=1.6$ close to the quantum critical point of the 2D KLM [58], where the RKKY interaction and Kondo screening are of the same order of magnitude, and thus it is crucial to treat them on equal footing. To this end, we use a finite-temperature version of the auxiliary-field quantum Monte Carlo (QMC) algorithm, as implemented in Ref. [59].

We consider the situation depicted in the inset of Fig. 1(a): starting with a single magnetic impurity deposited in the middle part of the metallic surface, in consecutive steps we add closed shells of magnetic moments 


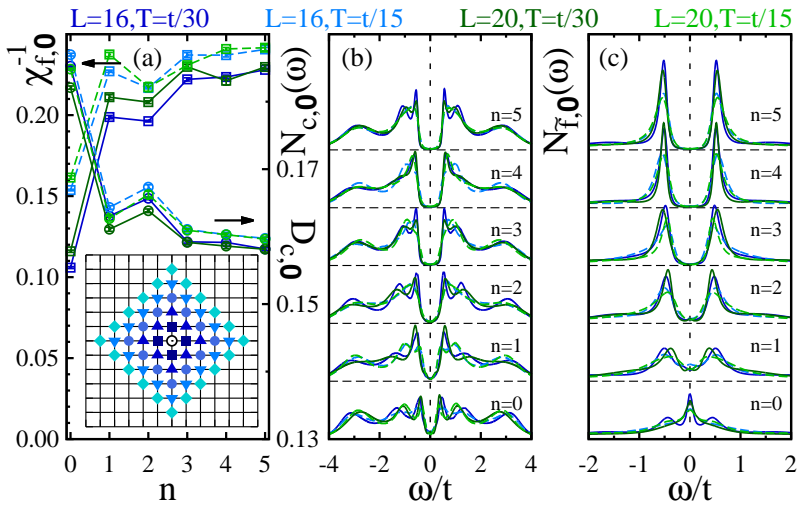

FIG. 1. Inverse $f$-spin susceptibility $\chi_{f, \mathbf{0}}^{-1}$ and $c$-electron double occupancy $D_{c, \mathbf{0}}(\mathrm{a}), c$-electron $\operatorname{LDOS} N_{c, \mathbf{0}}(\omega)$ (b), and $\tilde{f}$-operator LDOS $N_{\tilde{f}, \mathbf{0}}(\omega)$ (c) at the central site $\boldsymbol{r}=\mathbf{0}$ of a Kondo superlattice (see inset) upon increasing the number of impurity shells $n$ coupled to a $L \times L$ lattice of $c$-electrons at temperature $T$ much below the corresponding single-impurity Kondo scale $T_{K} \simeq t / 8$ [23]. Inset: construction of the superlattice; extra impurity shells around the initial impurity (open circle) are indicated using different symbols.

around the initial impurity so as to keep the coordination number fixed. This line of research has explicit experimental relevance: atomically precise engineering with STM was used to study the evolution of local density of states (LDOS) from an isolated iron(II) phthalocyanine molecule to the $2 \mathrm{D}$ superlattice on a $\mathrm{Au}(111)$ surface [60]. Although the underlying physics is complicated by the SU(4) Kondo effect [61], Ref. [60] provides a novel route to interpolate between the physics of a single Kondo impurity and the 2D Kondo lattice behavior.

First, we focus on local properties at the central impurity $\boldsymbol{r}=\mathbf{0}: \quad f$-spin susceptibility $\chi_{f, \boldsymbol{r}}=$ $\int_{0}^{\beta} d \tau\left\langle S_{f, \boldsymbol{r}}^{z}(\tau) S_{f, \boldsymbol{r}}^{z}(0)\right\rangle$ and $c$-electron double occupancy $D_{c, \boldsymbol{r}}=\left\langle n_{\boldsymbol{r}, \uparrow}^{c} n_{\boldsymbol{r}, \downarrow}^{c}\right\rangle$ with $n_{\boldsymbol{r}, \sigma}^{c}=c_{\boldsymbol{i}, \sigma}^{\dagger} c_{\boldsymbol{i}, \sigma}$, and discuss their evolution upon increasing number of impurity shells $n$. In a single-impurity problem, the susceptibility follows the Curie-Weiss law $\chi_{f} \propto 1 /(T+\Theta)$. As shown in Fig. 1(a), $\chi_{f, \mathbf{0}}^{-1}$ deviates rapidly from its single-impurity limit, shows an oscillating behavior, and finally starts to saturate in larger systems. Likewise, $D_{c, 0}$ shows a strong initial reduction and displays noticeable fluctuations before reaching saturation. The behavior of both $\chi_{f, \mathbf{0}}^{-1}$ and $D_{c, 0}$ suggests a fast onset of lattice effects.

The corresponding evolution of $c$-electron LDOS $N_{c, \mathbf{0}}(\omega)$ obtained from the imaginary-time-displaced Green's function $G_{c, \mathbf{0}}(\tau)=\sum_{\sigma}\left\langle c_{\mathbf{0}, \sigma}(\tau) c_{\mathbf{0}, \sigma}^{\dagger}(0)\right\rangle$ by means of the stochastic analytic continuation method [62] is shown in Fig. 1(b). While a precise form of the spectra is difficult to assess due to a complicated lineshape, the data reproducibly show that the narrow $\sim T_{K}$ dip at $\omega=0$ inherent to the single-impurity problem broadens and evolves into a full gap already in the $n=3$ system. A very natural account for the observed broadening is the RKKY interaction which is responsible for a broad quasiparticle gap $\sim J$ in the 2 D KLM $[58,63]$. Thus, our model QMC calculations rationalize the interpretation of spectroscopic characteristics in Ref. [60] in terms of the RKKY coupling between molecular spins in the central part of the superlattice.

The KLM forbids charge fluctuations on the $f$ orbitals. Instead, we examine a local spectral function $N_{\tilde{f}, \mathbf{0}}(\omega)$ extracted from the Green's function $G_{\tilde{f}, \mathbf{0}}(\tau)=$ $\sum_{\sigma}\left\langle\tilde{f}_{\mathbf{0}, \sigma}(\tau) \tilde{f}_{\mathbf{0}, \sigma}^{\dagger}(0)\right\rangle$, where the $\tilde{f}$-operator is defined as $\tilde{f}_{\mathbf{0}, \sigma^{\prime}}^{\dagger}=\sum_{\sigma}\left(c_{\mathbf{0}, \sigma}^{\dagger} f_{\mathbf{0}, \sigma} f_{\mathbf{0}, \sigma^{\prime}}^{\dagger}+f_{\mathbf{0}, \sigma^{\prime}}^{\dagger} c_{\mathbf{0}, \sigma}^{\dagger} f_{\mathbf{0}, \sigma}\right)$. The $\tilde{f}$ operator is derived from a single-impurity Anderson model (SIAM) using the Schrieffer-Wolff transformation $[64,65]$ and describes the so-called cotunelling process [66-68]: the tunelling of an electron from the STM tip into the conduction sea involves a spin-flip of the local magnetic $f$-moments. $A s$ is apparent, $N_{\tilde{f}, \mathbf{0}}(\omega)$ reproduces the Abrikosov-Suhl resonance of the SIAM in the Kondo model with a single impurity, see Fig. 1(c). Upon increasing $n$, the resonance splits first, then is replaced by a pseudogap, and finally a full gap opens up at $n=3$, coinciding with the gap that appears in $N_{c, 0}(\omega)$. While the splitting of the Abrikosov-Suhl resonance is already observed in the interimpurity spin-singlet state of the two-impurity Anderson model with a direct Heisenberg interaction between the $f$-spins [69-71], here we assume the absence of a direct overlap between the $f$ orbitals. Thus, the opening of the gap seems to signify the onset of Kondo coherence. Moreover, given that upon further increasing $n$ peaks on the flanks of the gap sharpen and become reminiscent of those found in the lattice limit $[20,72,73]$, it is tempting to assume that they stem from nearly flat HF bands with predominantly $f$-character.

Next, we consider the spatial properties of superlattices, summarized in Fig. 2. The build up of intersite AF spin correlations $S_{f}^{z}(\boldsymbol{r})=4\left\langle S_{f, \boldsymbol{r}}^{z} S_{f, \mathbf{0}}^{z}\right\rangle$ measured relative to the central impurity gives rise to a collective-like screening of the $f$-impurities, seen as the enhancement of $\chi_{f}^{-1}(\boldsymbol{r})$ at inner shells. It also assists the localization of $c$-electrons reflected in the reduction of $D_{c}(\boldsymbol{r})$ in the center. In contrast, the outermost shell, and in particular the corner sites, clearly stands out. It stems from two effects: (i) a reduced number of nearest-neighbor impurities at the edge makes the RKKY interaction less important and (ii) since the edge is immersed in a conduction electron sea, a locally enhanced density of $c$-electron states $\rho_{c, r}$ available for Kondo quenching of the edge impurities introduces a site-dependent Kondo temperature $T_{K}(\boldsymbol{r}) \sim e^{-1 / J \rho_{c, \boldsymbol{r}}(\omega=0)}$. Furthermore, while the spatial characteristics of the $n=3$ and larger superlattices are very much alike, cf. Figs. 2(g-i) and 1S in Ref. [65], one can discern a nonmonotonic evolution of both $\chi_{f}^{-1}(\boldsymbol{r})$ and 


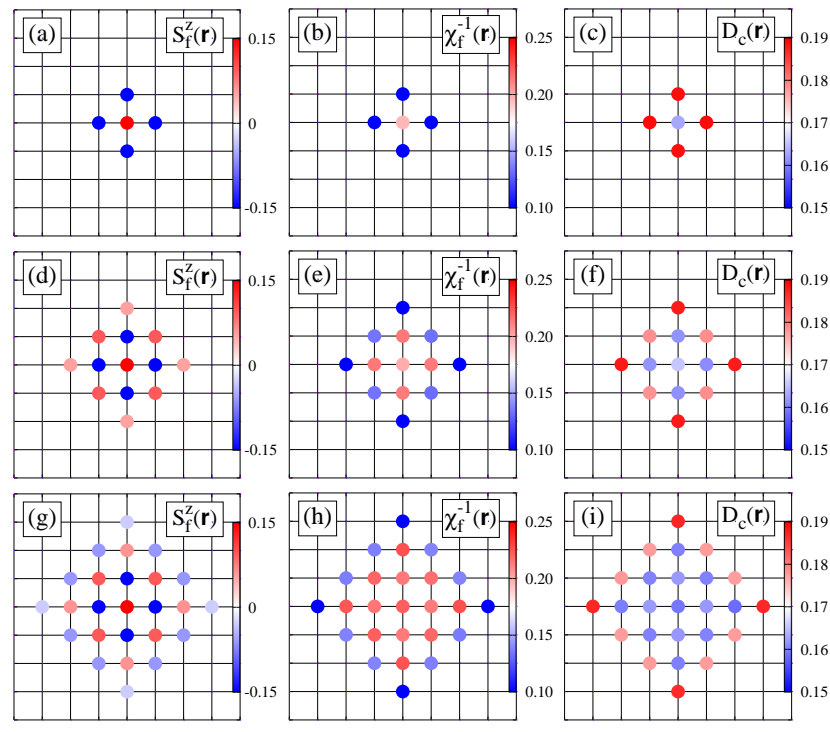

FIG. 2. Real-space spin correlations $S_{f}^{z}(\boldsymbol{r})$ relative to the central $\boldsymbol{r}=(0,0)$ impurity (left) and spatial dependence of: inverse $f$-spin susceptibility $\chi_{f}^{-1}(\boldsymbol{r})$ (middle) and $c$-electron double occupancy $D_{c}(\boldsymbol{r})$ (right) from QMC simulations of the $n=1$ (a-c), $n=2$ (d-f), and $n=3$ (g-i) systems. Parameters: $L=16$ and $T=t / 30$.

$D_{c}(\boldsymbol{r})$ when moving from the center towards the corner atom of the $n=2$ system. Together with the initial oscillating behavior of both quantities upon increasing $n$ in Fig. 1(a), it is indicative of a strong competition between the local Kondo physics at the edges and lattice effects in the center.

Given the onset of a Kondo insulating phase in the core of systems with $n \geq 3$, one would like to know what happens at the surface of the insulator immersed in a conduction electron sea, and in particular, whether and to what extent the insulator is penetrable to those electrons? To get more insight into this issue, we show in Fig. 3 how the $\tilde{f}$-operator LDOS $N_{\tilde{f}, \boldsymbol{r}}(\omega)$ evolves when moving from the central $(0,0)$ impurity to the corner $(n, 0)$ site for superlattices with different number of shells $n$. As is apparent, independently of whether the gap at the central site is full or partial, the corner always develops the Kondo peak, followed by the other sites of the outermost shell upon lowering $T$, see Fig. 3.

To assess that the emergence of low- $T$ Kondo peaks at the edge is not an artifact of analytical continuation, we focus on the largest $n=5$ nanosystem and look at the $T$-dependence of $N_{\tilde{f}, \boldsymbol{r}}(\omega=0)$, see the inset in Fig. 3 . It can be directly extracted from the Green's function $G_{\tilde{f}, \boldsymbol{r}}(\tau)$ by using $N_{\tilde{f}, \boldsymbol{r}}(\omega=0) \propto \lim _{\beta \rightarrow \infty} \beta G_{\tilde{f}, \boldsymbol{r}}(\tau=\beta / 2)$. As expected, all the site-dependent curves are bounded from below (above) by those corresponding to the 2D KLM [single-impurity Kondo model (SIKM)], respectively. The $T$-dependence of $N_{\tilde{f}, \boldsymbol{r}}(\omega=0)$ at the cen-
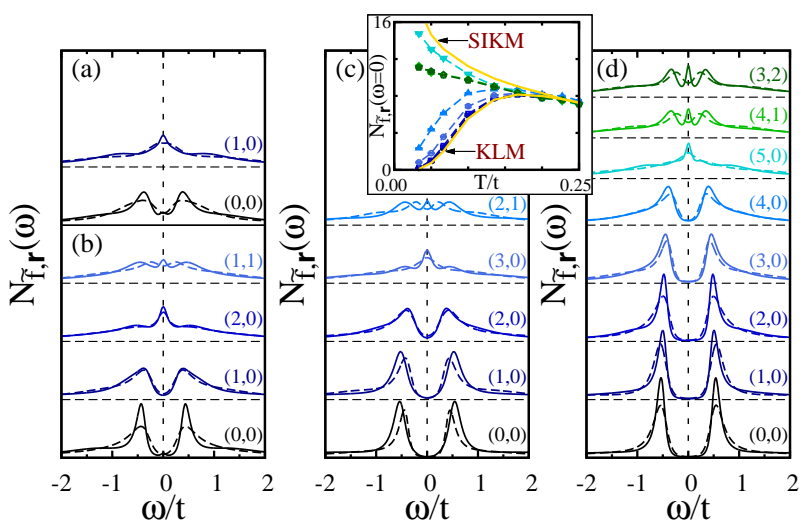

FIG. 3. Spatial variation (from bottom to top) of the $\tilde{f}$ operator LDOS $N_{\tilde{f}, \boldsymbol{r}}(\omega)$ along the $(0,0) \rightarrow(n, 0)$ (corner) path as well as at the corner-adjacent $(n-1,1)$ site of the outermost shell in the system with $n=1$ (a), $n=2$ (b), $n=3$ (c), and $n=5$ (d) shells. In (d) we also plot $N_{\tilde{f}, \boldsymbol{r}}(\omega)$ at the second inequivalent $(3,2)$ site of the outermost shell. Parameters: $L=20 ; T=t / 30$ (solid) and $T=t / 15$ (dashed). Inset: $T$ dependence of $N_{\tilde{f}, \boldsymbol{r}}(\omega=0)$ in the $n=5$ case with color-coding of sites as in panel (d); solid lines show two limiting cases: 2D KLM and the single-impurity Kondo model (SIKM).

tral impurity and at the two innermost $n \in\{1,2\}$ shells follows essentially that of the 2D KLM. Moreover, the $n \in\{3,4\}$ shells display a similar $T$-dependence indicative of the opening of full gap in the $T=0$ limit. In contrast, $N_{\tilde{f}, \boldsymbol{r}}(\omega=0)$ at the corner $(5,0)$ site follows closely the SIKM behavior; similarly, $N_{\tilde{f}, \boldsymbol{r}}(\omega=0)$ at the other $(4,1)$ and $(3,2)$ sites of the edge grows steadily with reducing $T$ lending further support for the emergent peaks at our lowest $T=t / 30$, see Fig. 3(d). They signal the penetration of conduction electron gas into the correlated superlattice via the Kondo effect and stem from the single-impurity Kondo physics, which prevails locally over intersite correlations. As such, they are specific to the geometry of a superlattice. Indeed, interfacing the Kondo lattice layer with a noninteracting metal leads merely to softening of the hybridization gap in the Kondo lattice layer [74].

An important issue that one might be concerned about is whether the opening of a direct (optical) gap in the local quantity $N_{\tilde{f}, r}(\omega)$ can be considered as unambiguous evidence of coherence, which is a global phenomenon in the system? Indeed, in a translation-invariant system, the appearance of a hybridized HF band structure with a small indirect gap is usually inferred either from the momentum-dependent single-particle spectral function $A(\boldsymbol{k}, \omega)[75-81]$ or from the $T$-dependence of transport properties [82, 83]. Given that the translational symmetry is broken in our superlattices, one has to use an alternative strategy to assess the formation of HF bands. One possibility stems from the fact that the 2D particlehole symmetric KLM subject to a magnetic field features 


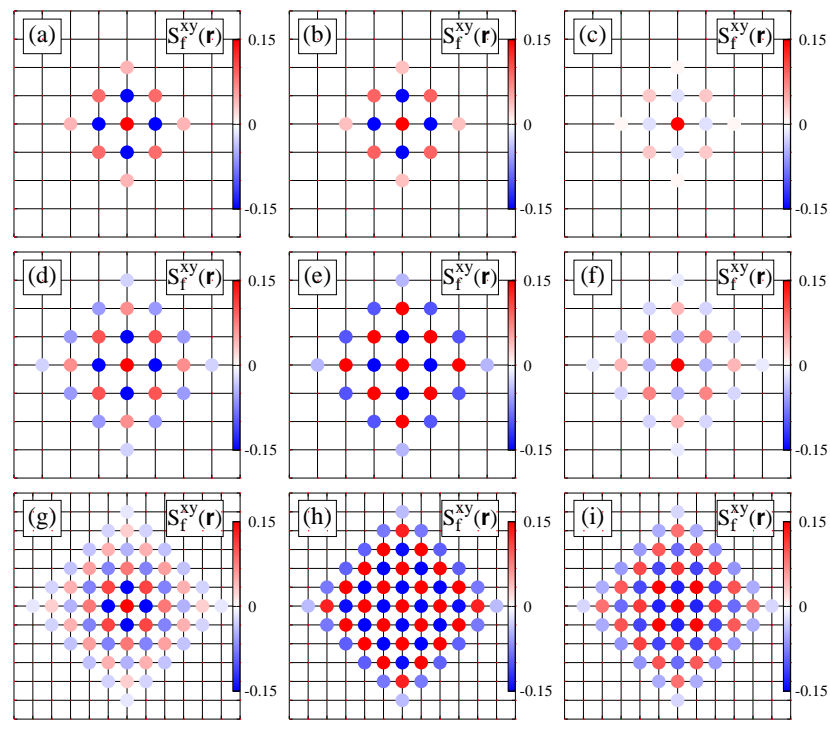

FIG. 4. Real-space transverse-spin correlations $S_{f}^{x y}(\boldsymbol{r})$ relative to the central impurity of the $n=2$ (a-c), $n=3$ (d-f), and $n=5$ (g-i) systems subject to increasing magnetic field $\mu_{B} B / t=0.06$ (left), 0.13 (middle), and 0.2 (right). Parameters: $L=16$ and $T=t / 30$.

a phase transition from the Kondo insulator to a canted AF state [84-86]. The AF phase is understood as a spindensity-wave instability driven by perfect nesting of the particle and hole hybridized bands with opposite spin indices. Thus, it is legitimate to consider the field-induced transverse antiferromagnetism as the criterion of the coherent Kondo lattice behavior.

With this considerations in mind, we perform QMC simulations of the KLM (1) augmented by a Zeeman term,

$$
H_{B}=-g \mu_{B} B \sum_{i}\left(S_{c, i}^{z}+S_{f, i}^{z}\right)
$$

and we calculate real-space transverse-spin correlations $S_{f}^{x y}(\boldsymbol{r})=2\left(\left\langle S_{f, \boldsymbol{r}}^{x} S_{f, \mathbf{0}}^{x}\right\rangle+\left\langle S_{f, \boldsymbol{r}}^{y} S_{f, \mathbf{0}}^{y}\right\rangle\right)$ relative to the central impurity upon increasing an external magnetic field $B$, see Fig. 4. The applied magnetic field quickly suppresses $S_{f}^{x y}(\boldsymbol{r})$ in the single-shell case, see Fig. $2 \mathrm{~S}$ in Ref. [65]. In the $n=2$ system, an enhanced range of finite $S_{f}^{x y}(\boldsymbol{r})$ in the field, albeit without any strengthening in Figs. 4(a-c), is evocative of the compensation effect: on the one hand, opening of the hybridization gap is a prerequisite for the emergence of nesting between the upper and lower HF bands with the opposite spin indices; on the other hand, enhanced quasiparticle scattering off transverse AF spin fluctuations in the presence of an incomplete gap, as in the $n=2$ superlattice, precludes the onset of coherence. In contrast, a full hybridization gap leads already in the $n=3$ system to a noticeable enhancement of transversespin correlations $S_{f}^{x y}(\boldsymbol{r})$, cf. Figs. 4(d,e), analogous to

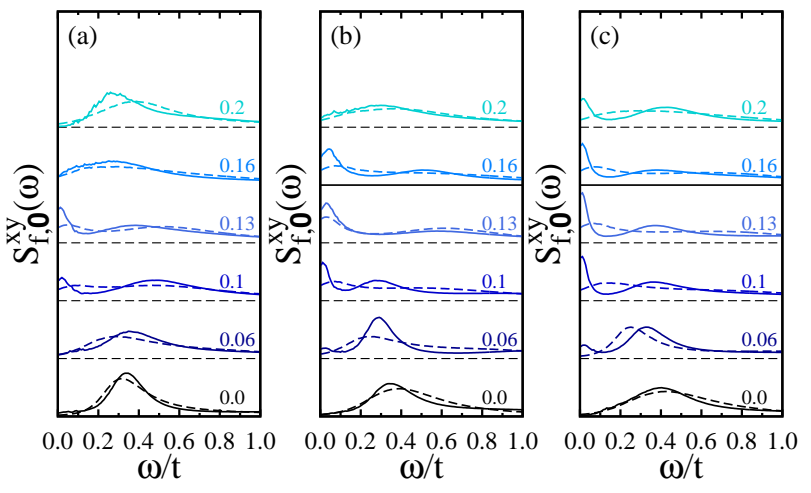

FIG. 5. Dynamical transverse-spin structure factor $S_{f, \mathbf{0}}^{x y}(\omega)$ at the central impurity as a function of out-of-plane magnetic field $B$ for the system with $n=2(\mathrm{a}), n=3$ (b), and $n=5$ (c) shells. From bottom to top: $\mu_{B} B / t=0, \ldots, 0.2$. Parameters: $L=16 ; T=t / 30$ (solid) and $T=t / 15$ (dashed).

that in the $n=5$ superlattice, see Figs. 4 (g-i). Same critical cluster size is found at smaller $J / t=1.5$ in the close proximity to the quantum critical point [Figs. $3 \mathrm{~S}(\mathrm{a}, \mathrm{b})$ in Ref. [65]] and at $J / t=1.8$ deep in the Kondo insulating phase [Figs. 3S(c,d) in Ref. [65]].

Finally, Fig. 5 displays how the corresponding dynamical transverse-spin structure factor $S_{f, \mathbf{0}}^{x y}(\omega)$ at the central site evolves as a function of the magnetic-field strength $B$. At $B=0$, all the spectra show a gap-like feature consistent with a collective screening of the $f$-impurities in the Kondo insulator. Increasing magnetic field results in a significant transfer of spectral weight to low-energy excitations. While the low-frequency spin-wave-like excitations are intimately connected to the field-induced transverse antiferromagnetism in the $n \geq 3$ systems, see Figs. 5(b,c), softening of spin excitations in the $n=2$ system in Fig. 5(a) should be considered as a precursive feature of the magnetic order that emerges in larger superlattices.

In summary, we have provided the evidence for the emergent coherent lattice behavior in molecular Kondo systems: despite a broken-translation symmetry by the surface, already a three-shell superlattice with $N_{\mathrm{imp}}=25$ periodically arranged magnetic impurities is sufficient to recover in the bulk features of the 2D periodic KLM. Our predictions are consistent with the recent studies of diluted Kondo lattice systems where disconnected $f$ electron clusters continue to exhibit the coherence temperature comparable to the clean case [87, 88]. The opening of a hybridization gap in the presence of the spin gap (Kondo insulator regime) effectively decouples the bulk from the metallic surface. This can be contrasted with zigzag graphene nanoribbons where the bulk is always metallic which leads to boundary-critical phenomena [89]. Our setup corresponds to a special band filling with exactly one conduction electron per impu- 
rity spin. It leads to the nesting-driven enhancement of the RKKY interaction, which assists the opening of the hybridization gap on top of that from coherent scattering off Kondo singlets. It motivates future QMC studies of the composite heavy quasiparticle formation in depleted Kondo nanosystems where some impurity spins are removed in a regular way, promoting HF metallicity $[90,91]$.

We thank Dirk K. Morr for helpful discussions and T. A. Costi for comments on the draft. M. R. was supported by the German Research Foundation (DFG) through Grant No. RA 2990/1-1. F. F. A. thanks the DFG for financial support through the SFB 1170 ToCoTronics (Project C01). The authors gratefully acknowledge the computing time granted by the John von Neumann Institute for Computing (NIC) and provided on the supercomputer JURECA [92] at Jülich Supercomputing Centre (JSC).

Note - A similar topic has recently been addressed in Ref. [93].

[1] F. Bloch, Über die Quantenmechanik der Elektronen in Kristallgittern, Z. Phys. 52, 555 (1929).

[2] S. Wirth and F. Steglich, Exploring heavy fermions from macroscopic to microscopic length scales, Nat. Rev. Mat. 1, 16051 (2016).

[3] A. C. Hewson, The Kondo Problem to Heavy Fermions (Cambridge University Press, Cambridge, 1993).

[4] T. Costi, E. Müller-Hartmann, and K. Ulrich, Quantum Monte Carlo simulations of dilute and concentrated heavy-fermion systems, Solid State Commun. 66, 343 (1988).

[5] P. Schlottmann, Impurity bands in Kondo insulators, Phys. Rev. B 46, 998 (1992).

[6] E. Miranda, V. Dobrosavljević, and G. Kotliar, DisorderDriven Non-Fermi-Liquid Behavior in Kondo Alloys, Phys. Rev. Lett. 78, 290 (1997).

[7] V. Barzykin and I. Affleck, Impurity correlations in dilute Kondo alloys, Phys. Rev. B 61, 6170 (2000).

[8] P. S. Riseborough, Collapse of the coherence gap in Kondo semiconductors, Phys. Rev. B 68, 235213 (2003).

[9] R. K. Kaul and M. Vojta, Strongly inhomogeneous phases and non-Fermi-liquid behavior in randomly depleted Kondo lattices, Phys. Rev. B 75, 132407 (2007).

[10] C. Grenzebach, F. B. Anders, G. Czycholl, and T. Pruschke, Influence of disorder on the transport properties of heavy-fermion systems, Phys. Rev. B 77, 115125 (2008).

[11] H. Watanabe and M. Ogata, Crossover from diluteKondo system to heavy-fermion system, Phys. Rev. B 81, 113111 (2010).

[12] P. Kumar and N. S. Vidhyadhiraja, Kondo-hole substitution in heavy fermions: Dynamics and transport, Phys. Rev. B 90, 235133 (2014).

[13] S. Sen, J. Moreno, M. Jarrell, and N. S. Vidhyadhiraja, Spectral changes in layered $f$-electron systems induced by Kondo hole substitution in the boundary layer, Phys. Rev. B 91, 155146 (2015).
[14] L.-y. Wei and Y.-f. Yang, Doping-induced perturbation and percolation in the two-dimensional Anderson lattice, Sci. Rep. 7, 46089 (2017).

[15] R. M. Martin, Fermi-Surfae Sum Rule and its Consequences for Periodic Kondo and Mixed-Valence Systems, Phys. Rev. Lett. 48, 362 (1982).

[16] C. Lacroix, Coherence effects in the Kondo lattice, J. Magn. Magn. Mater. 60, 145 (1986).

[17] Z. Tesanovic and O. T. Valls, Kondo lattice and the formation of a heavy-fermion state, Phys. Rev. B 34, 1918 (1986).

[18] M. A. Continentino, G. M. Japiassu, and A. Troper, Critical approach to the coherence transition in Kondo lattices, Phys. Rev. B 39, 9734 (1989).

[19] A. N. Tahvildar-Zadeh, M. Jarrell, and J. K. Freericks, Low-Temperature Coherence in the Periodic Anderson Model: Predictions for Photoemission of Heavy Fermions, Phys. Rev. Lett. 80, 5168 (1998).

[20] T. Pruschke, R. Bulla, and M. Jarrell, Low-energy scale of the periodic Anderson model, Phys. Rev. B 61, 12799 (2000).

[21] S. Burdin, A. Georges, and D. R. Grempel, Coherence Scale of the Kondo Lattice, Phys. Rev. Lett. 85, 1048 (2000).

[22] T. A. Costi and N. Manini, Low-Energy Scales and Temperature-Dependent Photoemission of Heavy Fermions, J. Low Temp. Phys. 126, 835 (2002).

[23] F. F. Assaad, Coherence scale of the two-dimensional Kondo lattice model, Phys. Rev. B 70, 020402 (2004).

[24] Y.-f. Yang, Z. Fisk, H.-O. Lee, J. D. Thompson, and D. Pines, Scaling the Kondo lattice, Nature (London) 454, 611 (2008).

[25] K. S. D. Beach and F. F. Assaad, Coherence and metamagnetism in the two-dimensional Kondo lattice model, Phys. Rev. B 77, 205123 (2008).

[26] M. Raczkowski, P. Zhang, F. F. Assaad, T. Pruschke, and M. Jarrell, Phonons and the coherence scale of models of heavy fermions, Phys. Rev. B 81, 054444 (2010).

[27] D. Tanasković, K. Haule, G. Kotliar, and V. Dobrosavljević, Phase diagram, energy scales, and nonlocal correlations in the Anderson lattice model, Phys. Rev. B 84, 115105 (2011).

[28] C. D. Bredl, S. Horn, F. Steglich, B. Lüthi, and R. M. Martin, Low-Temperature Specific Heat of $\mathrm{CeCu}_{2} \mathrm{Si}_{2}$ and $\mathrm{CeAl}_{3}$ : Coherence Effects in Kondo Lattice Systems, Phys. Rev. Lett. 52, 1982 (1984).

[29] Y. Ōnuki, Y. Shimizu, M. Nishihara, Y. Machii, and T. Komatsubara, Kondo Lattice Formation in $\mathrm{Ce}_{x} \mathrm{La}_{1-x} \mathrm{Cu}_{6}$, J. Phys. Soc. Jpn. 54, 1964 (1985).

[30] A. Sumiyama, Y. Oda, H. Nagano, Y. Ōnuki, K. Shibutani, and T. Komatsubara, Coherent Kondo State in a Dense Kondo Substance: $\mathrm{Ce}_{x} \mathrm{La}_{1-x} \mathrm{Cu}_{6}$, J. Phys. Soc. Jpn. 55, 1294 (1986).

[31] C. L. Lin, A. Wallash, J. E. Crow, T. Mihalisin, and P. Schlottmann, Heavy-fermion behavior and the singleion Kondo model, Phys. Rev. Lett. 58, 1232 (1987).

[32] S. L. Bud'ko, M. B. Fontes, and E. M. BaggioSaitovitch, Evolution of physical properties in the series $\mathrm{Ce}_{1-x} \mathrm{La}_{x} \mathrm{FeGe}_{3}$ : crossover from Kondo lattice to single ion Kondo impurity systems, J. Phys: Condens. Matter 10, 8815 (1998).

[33] S. Nakatsuji, S. Yeo, L. Balicas, Z. Fisk, P. Schlottmann, P. G. Pagliuso, N. O. Moreno, J. L. Sarrao, and J. D. Thompson, Intersite Coupling Effects in a Kondo Lattice, 
Phys. Rev. Lett. 89, 106402 (2002).

[34] A. P. Pikul, U. Stockert, A. Steppke, T. Cichorek, S. Hartmann, N. Caroca-Canales, N. Oeschler, M. Brando, C. Geibel, and F. Steglich, Single-Ion Kondo Scaling of the Coherent Fermi Liquid Regime in $\mathrm{Ce}_{1-x} \mathrm{La}_{x} \mathrm{Ni}_{2} \mathrm{Ge}_{2}$, Phys. Rev. Lett. 108, 066405 (2012).

[35] H. Hodovanets, S. L. Bud'ko, W. E. Straszheim, V. Taufour, E. D. Mun, H. Kim, R. Flint, and P. C. Canfield, Remarkably Robust and Correlated Coherence and Antiferromagnetism in $\left(\mathrm{Ce}_{1-x} \mathrm{La}_{x}\right) \mathrm{Cu}_{2} \mathrm{Ge}_{2}$, Phys. Rev. Lett. 114, 236601 (2015).

[36] D. Groten, G. J. C. v. Baarle, J. Aarts, G. J. Nieuwenhuys, and J. A. Mydosh, Thickness dependence of the ground-state properties of thin films of the heavy-fermion compound $\mathrm{CeCu}_{6}$, Phys. Rev. B 64, 144425 (2001).

[37] C. Echevarria-Bonet, D. P. Rojas, J. I. Espeso, J. Rodriguez Fernández, L. Rodriguez Fernández, E. Bauer, S. Burdin, S. G. Magalhães, and L. Fernández Barquin, Breakdown of the coherence effects and Fermi liquid behavior in $\mathrm{YbAl}_{3}$ nanoparticles, J. Phys.: Condens. Matter 30, 135604 (2018).

[38] M. Shimozawa, S. K. Goh, T. Shibauchi, and Y. Matsuda, From Kondo lattices to Kondo superlattices, Rep. Prog. Phys. 79, 074503 (2016).

[39] Y. Tada, R. Peters, and M. Oshikawa, Dimensional crossover in layered $f$-electron superlattices, Phys. Rev. B 88, 235121 (2013).

[40] M. Ternes, A. J. Heinrich, and W.-D. Schneider, Spectroscopic manifestations of the Kondo effect on single adatoms, J. Phys.: Condens. Matter 21, 053001 (2009).

[41] D. K. Morr, Theory of scanning tunneling spectroscopy: from Kondo impurities to heavy fermion materials, Rep. Prog. Phys. 80, 014502 (2017).

[42] V. Madhavan, W. Chen, T. Jamneala, M. F. Crommie, and N. S. Wingreen, Tunneling into a Single Magnetic Atom: Spectroscopic Evidence of the Kondo Resonance, Science 280, 567 (1998).

[43] J. Li, W.-D. Schneider, R. Berndt, and B. Delley, Kondo Scattering Observed at a Single Magnetic Impurity, Phys. Rev. Lett. 80, 2893 (1998).

[44] A. R. Schmidt, M. H. Hamidian, P. Wahl, F. Meier, A. V. Balatsky, J. D. Garrett, T. J. Williams, G. M. Luke, and J. C. Davis, Imaging the Fano lattice to hidden order transition in $\mathrm{URu}_{2} \mathrm{Si}_{2}$, Nature (London) 465, 570 (2010).

[45] S. Ernst, S. Kirchner, C. Krellner, C. Geibel, G. Zwicknagl, F. Steglich, and S. Wirth, Emerging local Kondo screening and spatial coherence in the heavy-fermion metal $\mathrm{YbRh}_{2} \mathrm{Si}_{2}$, Nature (London) 474, 362 (2011).

[46] M. H. Hamidian, A. R. Schmidt, I. A. Firmo, M. P. Allan, P. Bradley, J. D. Garrett, T. J. Williams, G. M. Luke, Y. Dubi, A. V. Balatsky, and J. C. Davis, How Kondo-holes create intense nanoscale heavy-fermion hybridization disorder, Proc. Natl. Acad. Sci. U.S.A. 108, 18233 (2011).

[47] P. Aynajian, E. H. da Silva Neto, A. Gyenis, R. E. Baumbach, J. D. Thompson, Z. Fisk, E. D. Bauer, and A. Yazdani, Visualizing heavy fermions emerging in a quantum critical Kondo lattice, Nature (London) 486, 201 (2012).

[48] L. Zhou, J. Wiebe, S. Lounis, E. Vedmedenko, F. Meier, S. Blügel, P. H. Dederichs, and R. Wiesendanger, Strength and directionality of surface Ruderman-KittelKasuya-Yosida interaction mapped on the atomic scale, Nat. Phys. 6, 187 (2010).
[49] H. Prüser, P. E. Dargel, M. Bouhassoune, R. G. Ulbrich, T. Pruschke, S. Lounis, and M. Wenderoth, Interplay between the Kondo effect and the Ruderman-Kittel-KasuyaYosida interaction, Nat. Commun. 5, 5417 (2014).

[50] A. Spinelli, M. Gerrits, R. Toskovic, B. Bryant, M. Ternes, and A. F. Otte, Exploring the phase diagram of the two-impurity Kondo problem, Nat. Commun. 6, 10046 (2015).

[51] A. Allerdt, C. A. Büsser, G. B. Martins, and A. E. Feiguin, Kondo versus indirect exchange: Role of lattice and actual range of $R K K Y$ interactions in real materials, Phys. Rev. B 91, 085101 (2015).

[52] A. K. Mitchell and R. Bulla, Validity of the local selfenergy approximation: Application to coupled quantum impurities, Phys. Rev. B 92, 155101 (2015).

[53] V. V. Savkin, A. N. Rubtsov, M. I. Katsnelson, and A. I. Lichtenstein, Correlated Adatom Trimer on a Metal Surface: A Continuous-Time Quantum Monte Carlo Study, Phys. Rev. Lett. 94, 026402 (2005).

[54] A. DiLullo, S.-H. Chang, N. Baadji, K. Clark, J.-P. Klckner, M.-H. Prosenc, S. Sanvito, R. Wiesendanger, G. Hoffmann, and S.-W. Hla, Molecular Kondo Chain, Nano Lett. 12, 3174 (2012).

[55] A. M. Lobos, M. A. Cazalilla, and P. Chudzinski, Magnetic phases in the one-dimensional Kondo chain on a metallic surface, Phys. Rev. B 86, 035455 (2012).

[56] A. K. Mitchell, P. G. Derry, and D. E. Logan, Multiple magnetic impurities on surfaces: Scattering and quasiparticle interference, Phys. Rev. B 91, 235127 (2015).

[57] H. Tsunetsugu, M. Sigrist, and K. Ueda, The groundstate phase diagram of the one-dimensional Kondo lattice model, Rev. Mod. Phys. 69, 809 (1997).

[58] F. F. Assaad, Quantum Monte Carlo Simulations of the Half-Filled Two-Dimensional Kondo Lattice Model, Phys. Rev. Lett. 83, 796 (1999).

[59] M. Bercx, F. Goth, J. S. Hofmann, and F. F. Assaad, The ALF (Algorithms for Lattice Fermions) project release 1.0. Documentation for the auxiliary field quantum Monte Carlo code, SciPost Phys. 3, 013 (2017).

[60] N. Tsukahara, S. Shiraki, S. Itou, N. Ohta, N. Takagi, and M. Kawai, Evolution of Kondo Resonance from a Single Impurity Molecule to the Two-Dimensional Lattice, Phys. Rev. Lett. 106, 187201 (2011).

[61] A. M. Lobos, M. Romero, and A. A. Aligia, Spectral evolution of the $S U(4)$ Kondo effect from the single impurity to the two-dimensional limit, Phys. Rev. B 89, 121406 (2014).

[62] K. S. D. Beach, Identifying the maximum entropy method as a special limit of stochastic analytic continuation, arXiv e-prints (2004), cond-mat/0403055.

[63] S. Capponi and F. F. Assaad, Spin and charge $d y$ namics of the ferromagnetic and antiferromagnetic twodimensional half-filled Kondo lattice model, Phys. Rev. B 63, 155114 (2001).

[64] J. R. Schrieffer and P. A. Wolff, Relation between the Anderson and Kondo Hamiltonians, Phys. Rev. 149, 491 (1966).

[65] See Supplemental Material, which includes Ref. [94], for a derivation of the $\tilde{f}$-operator and the additional numerical evidence of the emergent coherence in Kondo superlattices.

[66] M. Maltseva, M. Dzero, and P. Coleman, Electron Cotunneling into a Kondo Lattice, Phys. Rev. Lett. 103, 
206402 (2009).

[67] J. Figgins and D. K. Morr, Differential Conductance and Quantum Interference in Kondo Systems, Phys. Rev. Lett. 104, 187202 (2010).

[68] P. Wölfle, Y. Dubi, and A. V. Balatsky, Tunneling into Clean Heavy Fermion Compounds: Origin of the Fano Line Shape, Phys. Rev. Lett. 105, 246401 (2010).

[69] L. Zhu and J.-X. Zhu, Coherence scale of coupled Anderson impurities, Phys. Rev. B 83, 195103 (2011).

[70] T. Jabben, N. Grewe, and S. Schmitt, Spectral properties of the two-impurity Anderson model with varying distance and various interactions, Phys. Rev. B 85, 045133 (2012).

[71] L. Wang, H. Shinaoka, and M. Troyer, Fidelity Susceptibility Perspective on the Kondo Effect and Impurity Quantum Phase Transitions, Phys. Rev. Lett. 115, 236601 (2015).

[72] M. Vekić, J. W. Cannon, D. J. Scalapino, R. T. Scalettar, and R. L. Sugar, Competition between Antiferromagnetic Order and Spin-Liquid Behavior in the TwoDimensional Periodic Anderson Model at Half Filling, Phys. Rev. Lett. 74, 2367 (1995).

[73] N. S. Vidhyadhiraja, V. E. Smith, D. E. Logan, and H. R. Krishnamurthy, Dynamics and transport properties of Kondo insulators, J. Phys.: Condens. Matter 15, 4045 (2003).

[74] R. Peters, Y. Tada, and N. Kawakami, Kondo effect in f-electron superlattices, Phys. Rev. B 88, 155134 (2013).

[75] C. Gröber and R. Eder, Temperature-dependent band structure of the Kondo insulator, Phys. Rev. B 57, R12659 (1998).

[76] J. H. Shim, K. Haule, and G. Kotliar, Modeling the Localized-to-Itinerant Electronic Transition in the Heavy Fermion System CeIrIn 5 , Science 318, 1615 (2007).

[77] L. C. Martin and F. F. Assaad, Evolution of the Fermi Surface across a Magnetic Order-Disorder Transition in the Two-Dimensional Kondo Lattice Model: A Dynamical Cluster Approach, Phys. Rev. Lett. 101, 066404 (2008).

[78] J. Otsuki, H. Kusunose, and Y. Kuramoto, Evolution of a Large Fermi Surface in the Kondo Lattice, Phys. Rev. Lett. 102, 017202 (2009).

[79] A. Benlagra, T. Pruschke, and M. Vojta, Finitetemperature spectra and quasiparticle interference in Kondo lattices: From light electrons to coherent heavy quasiparticles, Phys. Rev. B 84, 195141 (2011).

[80] M. Klein, A. Nuber, H. Schwab, C. Albers, N. Tobita, M. Higashiguchi, J. Jiang, S. Fukuda, K. Tanaka, K. Shimada, M. Mulazzi, F. F. Assaad, and F. Reinert, Coherent Heavy Quasiparticles in a $\mathrm{CePt}_{5}$ Surface Alloy, Phys. Rev. Lett. 106, 186407 (2011).

[81] S.-K. Mo, W. S. Lee, F. Schmitt, Y. L. Chen, D. H. Lu, C. Capan, D. J. Kim, Z. Fisk, C.-Q. Zhang, Z. Hussain, and Z.-X. Shen, Emerging coherence with unified energy, temperature, and lifetime scale in heavy fermion $\mathrm{YbRh}_{2} \mathrm{Si}_{2}$, Phys. Rev. B 85, 241103 (2012).

[82] D. E. Logan and N. S. Vidhyadhiraja, Dynamics and transport properties of heavy fermions: theory, J. Phys.: Condens. Matter 17, 2935 (2005).

[83] C. Grenzebach, F. B. Anders, G. Czycholl, and T. Pruschke, Transport properties of heavy-fermion systems, Phys. Rev. B 74, 195119 (2006).

[84] K. S. D. Beach, P. A. Lee, and P. Monthoux, FieldInduced Antiferromagnetism in the Kondo Insulator,
Phys. Rev. Lett. 92, 026401 (2004).

[85] Milat, I., Assaad, F., and Sigrist, M., Field induced magnetic ordering transition in Kondo insulators, Eur. Phys. J. B 38, 571 (2004).

[86] T. Ohashi, A. Koga, S.-i. Suga, and N. Kawakami, Fieldinduced phase transitions in a Kondo insulator, Phys. Rev. B 70, 245104 (2004).

[87] N. C. Costa, T. Mendes-Santos, T. Paiva, N. J. Curro, R. R. dos Santos, and R. T. Scalettar, The Coherence Temperature in the Diluted Periodic Anderson Model, arXiv e-prints (2018), arXiv:1812.09426.

[88] M. Lawson, B. T. Bush, A. C. Shockley, C. Capan, Z. Fisk, and N. J. Curro, Site Specific Knight Shift Measurements of the Dilute Kondo lattice System $\mathrm{Ce}_{1-x} \mathrm{La}_{x} \mathrm{CoIn}_{5}$, arXiv e-prints (2019), arXiv:1901.01333.

[89] H. Karimi and I. Affleck, Towards a rigorous proof of magnetism on the edges of graphene nanoribbons, Phys. Rev. B 86, 115446 (2012).

[90] F. F. Assaad, Depleted Kondo lattices: Quantum Monte Carlo and mean-field calculations, Phys. Rev. B 65, 115104 (2002).

[91] N. C. Costa, M. V. Araújo, J. P. Lima, T. Paiva, R. R. dos Santos, and R. T. Scalettar, Compressible ferrimagnetism in the depleted periodic Anderson model, Phys. Rev. B 97, 085123 (2018).

[92] Jülich Supercomputing Centre, JURECA: Generalpurpose supercomputer at Jülich Supercomputing Centre, J. Large-Scale Res. Facil. 2, A62 (2016).

[93] J. Figgins, L. S. Mattos, W. Mar, Y.-T. Chen, H. C. Manoharan, and D. K. Morr, Quantum Engineered Kondo Lattices, arXiv e-prints (2019), arXiv:1902.04680.

[94] T. A. Costi, Kondo Effect in a Magnetic Field and the Magnetoresistivity of Kondo Alloys, Phys. Rev. Lett. 85, 1504 (2000). 


\section{Supplemental Material for: Emergent Coherent Lattice Behavior in Kondo Nanosystems}

\section{Schrieffer-Wolff transformation and electron cotunneling}

For simplicity we consider a symmetric single-impurity Anderson model (SIAM) defined as:

$$
\begin{aligned}
H_{\mathrm{SIAM}} & =\underbrace{\sum_{\boldsymbol{k}, \sigma} \varepsilon(\boldsymbol{k}) c_{\boldsymbol{k}, \sigma}^{\dagger} c_{\boldsymbol{k}, \sigma}+U\left(f_{\uparrow}^{\dagger} f_{\uparrow}-1 / 2\right)\left(f_{\downarrow}^{\dagger} f_{\downarrow}-1 / 2\right)}_{H_{0}} \\
& +\underbrace{\frac{V}{\sqrt{N}} \sum_{\boldsymbol{k}, \sigma}\left(c_{\boldsymbol{k}, \sigma}^{\dagger} f_{\sigma}+f_{\sigma}^{\dagger} c_{\boldsymbol{k}, \sigma}\right)}_{H_{1}} .
\end{aligned}
$$

The Abrikosov-Suhl resonance of the SIAM emerges in the single-particle spectral function of the $f$-electrons:

$$
G_{f}(\tau) \equiv \sum_{\sigma}\left\langle f_{\sigma}(\tau) f_{\sigma}^{\dagger}(0)\right\rangle=\int d \omega K(\tau, \omega) \operatorname{Im} G_{r e t}^{f}(\omega),
$$

where,

$$
\begin{aligned}
\operatorname{Im} G_{r e t}^{f}(\omega) & =\frac{\pi}{Z} \sum_{n, m, \sigma}\left(e^{-\beta E_{m}}+e^{-\beta E_{n}}\right) \\
& \times\left|\left\langle n\left|f_{\sigma}^{\dagger}\right| m\right\rangle\right|^{2} \delta\left(\omega+E_{n}-E_{m}\right),
\end{aligned}
$$

and the Kernel is given by:

$$
K(\tau, \omega)=\frac{1}{\pi} \frac{e^{\tau \omega}}{1+e^{\beta \omega}} .
$$

We now carry out the Schrieffer-Wolff canonical transformation required to eliminate the hybridization term in first order and to derive the Kondo model. Let

$$
S^{\dagger}=-S, \text { and }\left[S, H_{0}\right]=-H_{1},
$$

then

$$
e^{S} H e^{-S}=H_{0}+\frac{1}{2}\left[S, H_{1}\right]+\mathcal{O}\left(\epsilon^{3}\right) .
$$

Here, we have formally assumed that $H_{1}$ and the generator $S$ are of order $\epsilon$. Under this canonical transformation, the imaginary-time-displaced Green's function:

$$
G_{\tilde{f}}(\tau) \equiv \sum_{\sigma}\langle\underbrace{e^{S} f_{\sigma} e^{-S}}_{\tilde{f}_{\sigma}}(\tau) \underbrace{e^{S} f_{\sigma}^{\dagger} e^{-S}}_{\tilde{f}_{\sigma}^{\dagger}}(\tau=0)\rangle,
$$

should reproduce the Abrikosov-Suhl resonance of the SIAM in the Kondo model with a single impurity. The calculation gives:

$$
\begin{aligned}
S & =\frac{V}{\sqrt{N}} \sum_{\boldsymbol{k}, \sigma} \\
& \left(\frac{c_{\boldsymbol{k}, \sigma}^{\dagger} f_{\sigma} P_{o}}{\varepsilon(\boldsymbol{k})+U / 2-i 0^{+}}+\frac{c_{\boldsymbol{k}, \sigma}^{\dagger} f_{\sigma} P_{e}}{\varepsilon(\boldsymbol{k})-U / 2-i 0^{+}}-H . c .\right)
\end{aligned}
$$

where $P_{e}\left(P_{o}\right)$ is the projection of the even, $(-1)^{\sum_{\sigma} f_{\sigma}^{\dagger} f_{\sigma}}=1$ (odd, $(-1)^{\sum_{\sigma} f_{\sigma}^{\dagger} f_{\sigma}}=-1$ ), respectively, parity sector of the $f$-electron. We can now transform the creation operator for $f$-electrons, and constrain the Hilbert space to $P_{o}$ so as to obtain:

$$
\begin{aligned}
\tilde{f}_{\sigma^{\prime}}^{\dagger} & \equiv e^{S} f_{\sigma^{\prime}}^{\dagger} e^{-S} \\
& \simeq \frac{V}{\sqrt{N}} \sum_{\boldsymbol{k}, \sigma}\left(\frac{c_{\boldsymbol{k}, \sigma}^{\dagger} f_{\sigma} f_{\sigma^{\prime}}^{\dagger}}{\varepsilon(\boldsymbol{k})-U / 2-i 0^{+}}-\frac{f_{\sigma^{\prime}}^{\dagger} c_{\boldsymbol{k}, \sigma}^{\dagger} f_{\sigma}}{\varepsilon(\boldsymbol{k})+U / 2-i 0^{+}}\right)
\end{aligned}
$$

The first (second) term involves a virtual doubly (empty) occupied $f$-site. Assuming that $U$ is the largest scale so that we can set $\varepsilon(\boldsymbol{k})$ to zero gives the final result:

$$
\tilde{f}_{\sigma^{\prime}}^{\dagger} \simeq-\frac{V}{U} \sum_{\sigma}\left(c_{\boldsymbol{r}=\mathbf{0}, \sigma}^{\dagger} f_{\sigma} f_{\sigma^{\prime}}^{\dagger}+f_{\sigma^{\prime}}^{\dagger} c_{\boldsymbol{r}=\mathbf{0}, \sigma}^{\dagger} f_{\sigma}\right) .
$$

Using the relation, $2 f_{\sigma^{\prime}}^{\dagger} f_{\sigma^{\prime}}=n_{f}+\sigma^{\prime} 2 S_{f}^{z}$, where $\sigma^{\prime}$ takes the value $1(-1)$ for up (down) spin degrees of freedom, $S_{f}^{z}=\frac{1}{2} \sum_{\sigma^{\prime}} \sigma^{\prime} f_{\sigma^{\prime}}^{\dagger} f_{\sigma^{\prime}}$, and $n_{f}=\sum_{\sigma^{\prime}} f_{\sigma^{\prime}}^{\dagger} f_{\sigma^{\prime}}$, the above operator can be rewritten as:

$$
\tilde{f}_{\sigma^{\prime}}^{\dagger} \simeq \frac{2 V}{U}\left(c_{\boldsymbol{r}=\mathbf{0},-\sigma^{\prime}}^{\dagger} S_{f}^{\sigma^{\prime}}+\sigma^{\prime} c_{\boldsymbol{r}=\mathbf{0}, \sigma^{\prime}}^{\dagger} S_{f}^{z}\right)
$$

Here $S_{f}^{\sigma^{\prime}}=f_{\sigma^{\prime}}^{\dagger} f_{-\sigma^{\prime}}$ and we have used the fact that in the Kondo regime $n_{f}=1$. The corresponding impurity spectral function for the Kondo model matches that derived in Ref. [94] using the equation of motion for the c-electron Green's function.

\section{Supplemental data}

TABLE I. Total number of magnetic impurities $N_{\text {imp }}$ in a nanosystem with $n$ shells.

\begin{tabular}{lllcccc}
\hline \hline$n$ & 0 & 1 & 2 & 3 & 4 & 5 \\
$N_{\text {imp }}$ & 1 & 5 & 13 & 25 & 41 & 61 \\
\hline \hline
\end{tabular}

The relation between the number of shells $n$ and the corresponding total number of impurities $N_{\text {imp }}$ in a superlattice is summarized in Table I. Figure 1S shows the spatial characteristics of the $n \in\{4,5\}$ superlattices. The enhancement of transverse-spin correlations $S_{f}^{x y}(\boldsymbol{r})$ for $n \geq 3$ upon increasing the external magnetic field $B$ at $J / t=1.6$ is documented more quantitatively in Fig. $2 \mathrm{~S}$. Same critical cluster size required to observe the enhancement of $S_{f}^{x y}(\boldsymbol{r})$ is found at smaller $J / t=1.5$ in the close proximity to the quantum critical point [Figs. 3S(a,b)] and at $J / t=1.8$ deep in the Kondo insulating phase [Figs. 3S $(\mathrm{c}, \mathrm{d})]$. 

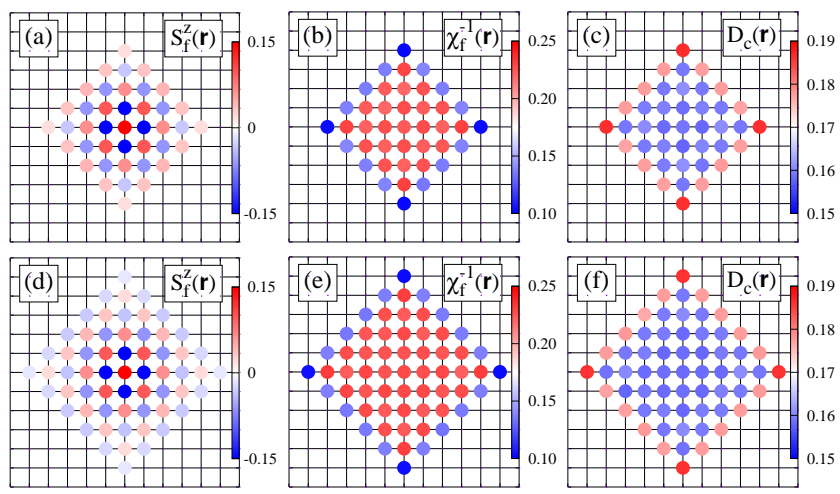

FIG. 1S. Real-space spin correlations $S_{f}^{z}(\boldsymbol{r})$ relative to the central impurity (left) and spatial dependence of: inverse $f$-spin susceptibility $\chi_{f}^{-1}(\boldsymbol{r})$ (middle) and $c$-electron double occupancy $D_{c}(\boldsymbol{r})$ (right) in the $n=4$ (a-c) and $n=5$ (d-f) systems. Parameters: $J / t=1.6, T=t / 30$, and $L=16$.

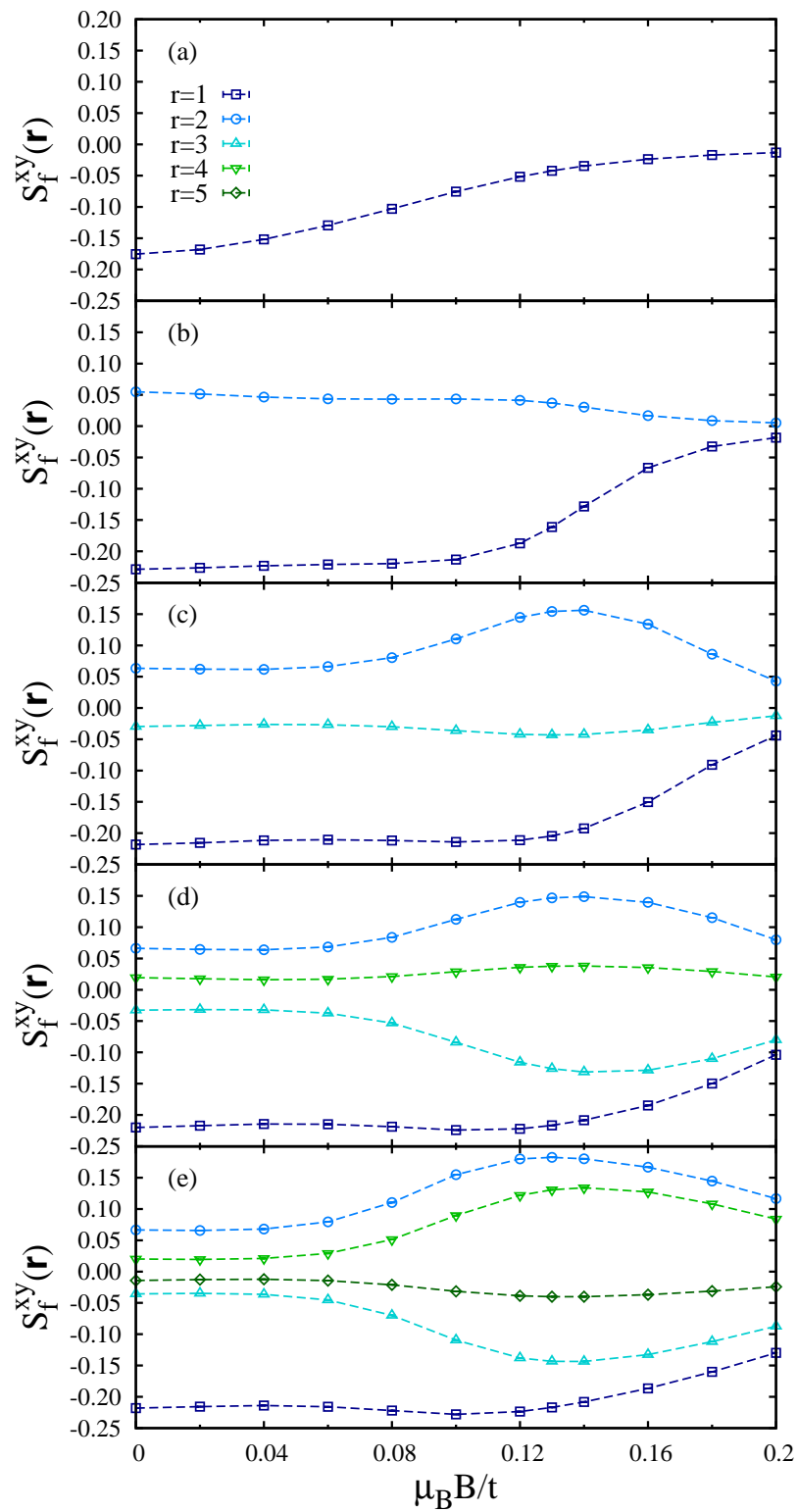

FIG. 2S. Transverse-spin correlations $S_{f}^{x y}(\boldsymbol{r})$ between the $(i, 0)$ site with $i=1, \ldots, n$ and the central $(0,0)$ impurity as a function of ouf-of-plane magnetic field $B$ for the system with $n=1$ (a), $n=2$ (b), $n=3$ (c), $n=4$ (d), and $n=5$ (e) shells. Parameters as in Fig. 1S. 


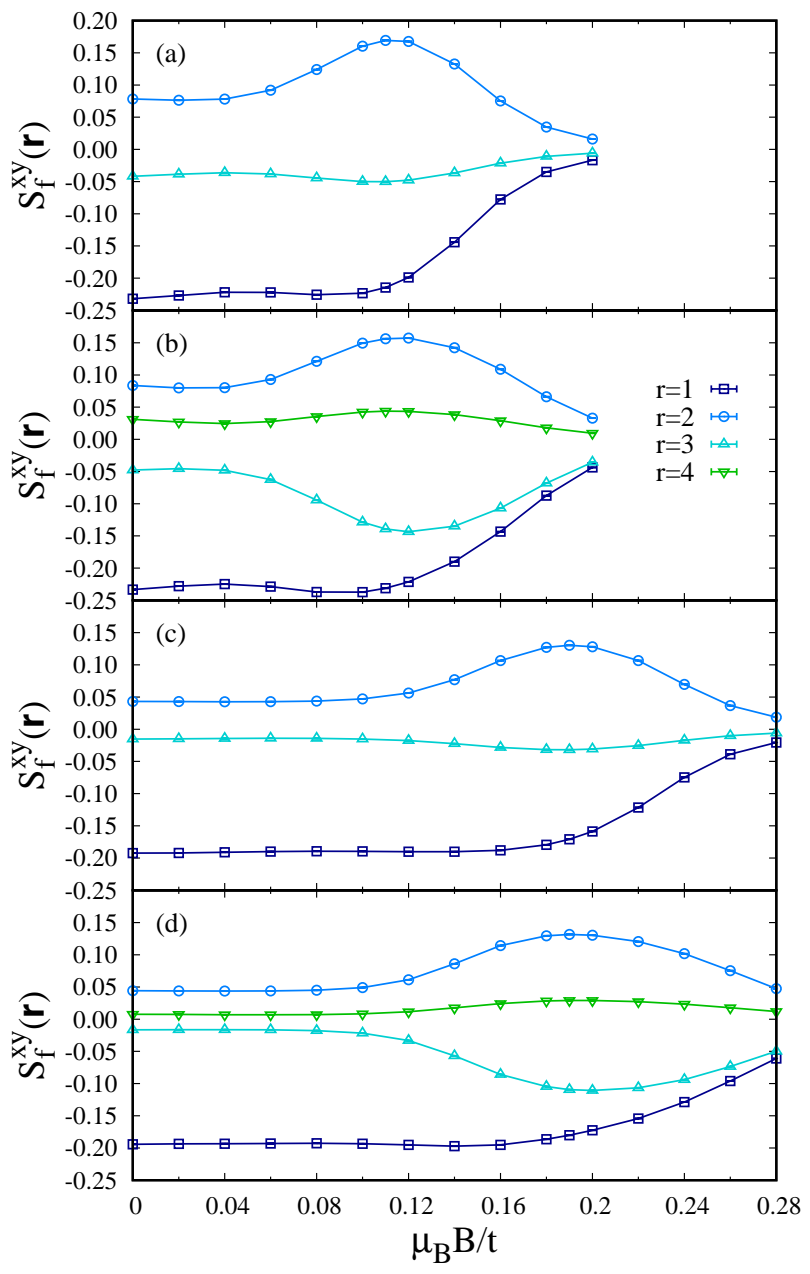

FIG. 3S. Same as in Fig. $2 \mathrm{~S}$ but for $J / t=1.5$ with $n=3$ (a) and $n=4$ (b) and for $J / t=1.8$ with $n=3$ (c) and $n=4$ (d). 